\title{
Convergence of Ishikawa type iteration process for three quasi-nonexpansive mappings in a convex metric space
}

\author{
Hafiz Fukhar-ud-din
}

\begin{abstract}
The paper establishes some convergence theorems for Ishikawa type iteration processes associated with two and three quasi-nonexpansive mappings in a convex metric space.
\end{abstract}

\section{Introduction}

The Banach contraction principle asserts that a contraction on a complete metric space has a unique fixed point and its proof hinges on "Picard iterations". This principle is applicable to a variety of subjects such as integral equations, partial differential equations and engineering of image processing. This principle fails for nonexpansive mappings even on a Banach space. Mann [Mean value methods in iterations, Proc. Amer. Math. Soc. 4 (1953), 506-510] introduced an iterative scheme to approximate fixed points of a nonexpansive mapping on a Banach space. This scheme breakes down for pseudocontractive mappings on a Hilbert space. Consequently, Ishikawa [Fixed points by a new iteration method, Proc. Amer. Math.Soc., 44 (1974), 147-150] upgraded Mann iterative scheme. Mann and Ishikawa iterative processes for nonexpansive and quasi-nonexpansive mappings have been extensively studied in uniformly convex Banach spaces and Hilbert spaces $[1,4,5,8,10,11,16]$. Das and Debata [6] studied strong convergence of Ishikawa iterates $\left\{x_{n}\right\}$ defined by

Key Words: Uniformly convex metric space, Common fixed point, Quasi-nonexpansive mapping, Conditions I-II, Strong convergence.

2010 Mathematics Subject Classification: 47H09,47H10, 47J25.

Received: January, 2014.

Revised: May, 2014.

Accepted: May, 2014. 


$$
x_{1} \in C, x_{n+1}=\alpha_{n} S\left[\beta_{n} T x_{n}+\left(1-\beta_{n}\right) x_{n}\right]+\left(1-\alpha_{n}\right) x_{n}
$$

for two quasi-nonexpansive mapings in a strictly convex Banach space. Nonlinear version of a known problem on a linear domain, is of great importance. In the literature, several mathematicians have introduced the notion of convexity in metric spaces; (see, for example, Kirk [14, 15], Penot [18] and Takahashi [20]).

A convex structure [20], in a metric space $(X, d)$ has been introduced by Takahashi [20] as a mapping $W: X^{2} \times[0,1] \rightarrow X$ satisfying the inequality

$$
d(u, W(x, y, \alpha)) \leq \alpha d(u, x)+(1-\alpha) d(u, y)
$$

for all $x, y, u \in X$ and $\alpha \in I=[0,1]$. The metric space $(X, d)$ together with a convex structure $W$ is known as a convex metric space. A nonempty subset $C$ of $X$ is convex if $W(x, y, \alpha) \in C$ for all $x, y \in C$ and $\alpha \in I$. Hadamard manifolds [3] and $C A T(0)$ spaces [2, 13] are nonlinear examples of convex metric spaces.

A convex metric space $X$ is said to satisfy Property $(\mathrm{H})$ [7] if

$$
d(W(x, y, \alpha), W(z, y, \alpha)) \leq \alpha d(x, z) \text { for all } x, y, z \in X \text { and } \alpha \in I \text {. }
$$

A convex metric space $X$ is strictly convex[20] if for any $x, y \in X$ and $\alpha \in$ $I$, there exists a unique element $z \in X$ such that $\alpha d(x, y)=d(z, y)$ and $(1-\alpha) d(x, y)=d(x, z)$ and uniformly convex [19] if for any $\varepsilon>0$, there exists $\alpha_{\varepsilon}>0$ such that $d\left(z, W\left(x, y, \frac{1}{2}\right)\right) \leq r\left(1-\alpha_{\varepsilon}\right)<r$ for all $r>0$ and $x, y, z \in X$ with $d(z, x) \leq r, d(z, y) \leq r$ and $d(x, y) \geq r \varepsilon$.

Let $C$ be a nonempty subset of a metric space. A mapping $T: C \rightarrow C$ is: (i) nonexpansive if $d(T x, T y) \leq d(x, y)$ for all $x, y \in C$ (ii) quasi-nonexpansive if for any $p \in C$ and $T p=p$, we have $d(T x, p) \leq d(x, p)$ for all $x \in C$.

Quasi-nonexpansive mappings are more general than nonexpansive mappings. A nonexpansive mapping with at least one fixed point is quasi-nonexpansive.

We give the following examples.

Example 1.1. Define a mapping $T:[0,2] \rightarrow[0,2]$ by $T x=0$ if $x \neq 2$ and $T x=1$ if $x=2$.Then $T$ is quasi-nonexpansive but not continuous under usual norm on the set of reals.

Proof. Obviously 0 is the only fixed point of $T$ and $|T x-T 0|=|T x| \leq|x|=$ $|x-0|$ for all $x \in[0,2]$. Therefore $T$ is quasi-nonexpansive. $T$ is not continuous because $\left\{2-\frac{1}{n}\right\} \subset[0,2]$ and $2-\frac{1}{n} \rightarrow 2$ but $T\left(2-\frac{1}{n}\right)=0 \nrightarrow 1=T 2$. 
Example 1.2. Define $T:[-1,1] \rightarrow[-1,1]$ by $T x=\frac{2 x}{3} \sin \frac{1}{x}$ if $x \neq 0$ and $T x=0$ if $x=0$.Then $T$ is continuous and quasi-nonexpansive but not nonexpansive under usual norm on the set of reals.

Proof. Obviously $T$ is continuous and 0 is the only fixed point of $T$.If $x \neq 0$ and $T x=x$, then $x=\frac{2 x}{3} \sin \frac{1}{x}$ or $\sin \frac{1}{x}=\frac{3}{2}$ for $x \in[-1,0) \cup(0,1]$ which is impossible. Since $|T x-0|=\frac{2|x|}{3}\left|\sin \frac{1}{x}\right| \leq \frac{2|x|}{3}$. Therefore $|T x-0|<$ $|x-0|$ for all $x \in[-1,1]$. Hence $T$ is quasi-nonexpansive. However, $T$ is not nonexpansive mapping. This is confirmed by choosing $x=\frac{2}{\pi}, y=\frac{2}{3 \pi}$. Then $|T x-T y|=\frac{2}{3}\left|\frac{2}{\pi} \sin \frac{\pi}{2}-\frac{2}{3 \pi} \sin \frac{3 \pi}{2}\right|=\frac{2}{3}\left(\frac{2}{\pi}+\frac{2}{3 \pi}\right)=\frac{4}{3 \pi}\left(1+\frac{1}{3}\right)=\frac{16}{9 \pi}$, $|x-y|=\left|\frac{2}{\pi}-\frac{2}{3 \pi}\right|=\frac{4}{3 \pi}$ and $\frac{16}{9 \pi}>\frac{4}{3 \pi}$.

Suppose $C$ is a convex subset of $X$ and $S, T$ are two self mappings on $C$. For $x_{1} \in C$, we define a sequence $\left\{x_{n}\right\}$ by

$$
\begin{aligned}
x_{n+1} & =W\left(T y_{n}, S x_{n}, \alpha_{n}\right), \\
y_{n} & =W\left(T x_{n}, S x_{n}, \beta_{n}\right), \quad n \geq 1,
\end{aligned}
$$

where $\alpha_{n}, \beta_{n}$ satisfy (i) $0<a \leq \alpha_{n} \leq b<1$ and (ii) $0 \leq \beta_{n} \leq \beta<1$. If $S=I$ (the identity mapping), the iterates in (2) become Ishikawa iterates for one mapping in a convex metric space.

Denote the set of fixed points of $T$ by $F(T)$.

Two mappings $S, T: C \rightarrow C$ satisfy Condition (I) if there exists a nondecreasing function $f:[0, \infty) \rightarrow[0, \infty)$ with $f(0)=0$ and $f(r)>0$ for $r>0$ such that $d(S x, T y) \geq f(d(x, F(S, T)))$ for $x, y \in C$ with $y=W(T x, S x, t)$, where $0 \leq t \leq \beta<1, F(S, T)$ is the set of common fixed points of $S$ and $T$ and $d(x, F(S, T))=\inf \{d(x, y): y \in F(S, T)\}$.

If we set $S=I$ in Condition (I), it becomes Condition 3 of Khan et. al. ([12], p.3) and an analogue of the Condition (A) of Maiti and Ghosh [16] in normed spaces.

In this paper, we define and study an itetation process for two and three quasi-nonexpansive mappings on a nonlinear domain, namely, a uniformly convex metric space and prove its convergence to common fixed point of the mappings under weaker assumptions. The results established in this paper, in particular, hold for uniformly convex Banach spaces and $C A T(0)$ spaces, simultaneously.

In the sequel, the following results are needed.

Lemma 1.3. [20]Let $X$ be a convex metric space. Then the open sphere $S(x, r)=\{y \in X: d(y, x)<r\}$ and the closed sphere $S[x, r]=$ $=\{y \in X: d(y, x) \leq r\}$ are convex subsets of $X$. 
Lemma 1.4. [19] Every bounded decreasing sequence of nonempty, closed and convex subsets of a complete uniformly convex metric space $X$, has a nonempty intersection.

Lemma 1.5. [11]Let $X$ be a uniformly convex metric space satisfying property (H). Let $x \in X$ and $\left\{\alpha_{n}\right\}$ be a sequence in $[b, c]$ for some $b, c \in(0,1)$. If $\left\{u_{n}\right\}$ and $\left\{v_{n}\right\}$ are sequences in $X$ such that $\limsup _{n \rightarrow \infty} d\left(u_{n}, x\right) \leq r$,

$\limsup \sup _{n \rightarrow \infty} d\left(v_{n}, x\right) \leq r$ and $\lim _{n \longrightarrow \infty} d\left(W\left(u_{n}, v_{n}, \alpha_{n}\right), x\right)=r$ for some $r \geq 0$, then $\lim _{n \rightarrow \infty} d\left(u_{n}, v_{n}\right)=0$.

\section{Convergence Analysis}

Lemma 2.1. Let $C$ be a nonempty subset of a strictly convex metric space $X$ and $T$ be a quasi -nonexpansive self mapping on $C$. Then $F(T)$ is closed on which $T$ is continuous.

Proof. Let $x$ be a limit point of $F(T)$ and $x \notin F(T)$. Let $r=\frac{1}{3} d(x, T x)>0$. Since $x$ is a limit point of $F(T)$, so there exists $y \in F(T)$ such that $d(x, y)<$ $r$.Since $T$ is quasi-nonexpansive, we have

$$
d(T x, y) \leq d(x, y)<r .
$$

So we have

$$
3 r=d(x, T x) \leq d(x, y)+d(T x, y)<2 r .
$$

This contradiction establishes that $F(T)$ is closed.

Let $x_{n}, x \in F(T)$ such that $x_{n} \rightarrow x$. So by definition of $F(T), T\left(x_{n}\right) \rightarrow$ $T(x)$ which shows that $T$ is continuous on $F(T)$.

Theorem 2.2. Let $C$ be a nonempty, closed and convex subset of a uniformly convex complete metric space $X$ satisfying property $(H)$. If $S$ and $T$ are quasinonexpansive self mappings on $C$ and satisfy Condition (I), then the sequence $\left\{x_{n}\right\}$ in (1.2), converges to an element of $F(S, T)$.

Proof. Define $C_{1}=\{y \in X: d(y, p) \leq r\} \cap C$ where $r=d\left(x_{1}, p\right)$ for some $x_{1} \in C$ and $p \in F(S, T)$. Then $C_{1}$ is a nonempty, closed and convex subset of $C$. Both $T x$ and $S x$ are in $C$ for $x \in C$. Therefore $C_{1}$ is invarient under $S$ and $T$. Without loss of generality, we assume that $C$ is bounded. For $p \in F(S, T)$, the routine calculations give that

$$
d\left(x_{n+1}, p\right) \leq d\left(x_{n}, p\right) \text { for all } n \geq 1 .
$$


Hence $\lim _{n \rightarrow \infty} d\left(x_{n}, p\right)=c$ (say) and assume that $c>0$, otherwise the conclusion is obvious.

Obviously,

$$
\limsup _{n \rightarrow \infty} d\left(T x_{n}, p\right) \leq c
$$

and

$$
\limsup _{n \rightarrow \infty} d\left(S y_{n}, p\right) \leq c .
$$

Moreover, $\lim _{n \rightarrow \infty} d\left(x_{n+1}, p\right)=c$ gives that

$$
\lim _{n \rightarrow \infty} d\left(W\left(T y_{n}, S x_{n}, \alpha_{n}\right), p\right)=c .
$$

In the light of (3)-(5), we apply Lemma 1.5 and get that $\lim _{n \rightarrow \infty} d\left(T y_{n}, S x_{n}\right)=$ 0 . Now, with the help of Condition (I), we have that $\lim _{n \rightarrow \infty} d\left(x_{n}, F(S, T)\right)=$ 0 . For a given $\varepsilon>0$, there exists $N_{\varepsilon} \geq 1$ and $y_{\varepsilon} \in F(S, T)$ such that $d\left(x_{n}, y_{\varepsilon}\right)<\varepsilon$ for all $n \geq N_{\varepsilon}$.Thus, if $\varepsilon_{k}=2^{-k}$ for $k \geq 1$, then corresponding to each $\varepsilon_{k}$, there exist $N_{k} \geq 1$ and $y_{k} \in F(S, T)$ such that $d\left(x_{n}, y_{k}\right) \leq \frac{\varepsilon_{k}}{4}$ for all $n \geq N_{k}$. On choosing $N_{k+1} \geq N_{k}$ for any $k \geq 1$,we have that

$$
\begin{aligned}
d\left(y_{k}, y_{k+1}\right) & \leq d\left(y_{k}, x_{N_{k+1}}\right)+d\left(x_{N_{k+1}}, y_{k+1}\right) \\
& <\frac{\varepsilon_{k}}{4}+\frac{\varepsilon_{k+1}}{4}=\frac{\varepsilon_{k}}{4}\left(1+\frac{1}{2}\right)=\frac{3}{4} \varepsilon_{k+1} .
\end{aligned}
$$

If $x \in S\left[y_{k+1}, \varepsilon_{k+1}\right]$, then

$$
\begin{aligned}
d\left(x, y_{k}\right) & \leq d\left(x, y_{k+1}\right)+d\left(y_{k+1}, y_{k}\right) \\
& <\varepsilon_{k+1}+\frac{3}{4} \varepsilon_{k+1}=\frac{7}{4} \varepsilon_{k+1}<2 \varepsilon_{k+1}=\varepsilon_{k}
\end{aligned}
$$

which gives that $x \in S\left[y_{k}, \varepsilon_{k}\right]$.Hence $\left\{S\left[y_{k}, \varepsilon_{k}\right]: k \geq 1\right\}$ is a decreasing sequence of nonempty, bounded, closed and convex subsets in a uniformly convex complete metric space and so $\cap_{k=1}^{\infty} S\left[y_{k}, \varepsilon_{k}\right] \neq \emptyset$ by Lemma 1.4. Now there exists a $p \in X$ such that

$$
d\left(y_{k}, p\right) \leq \frac{1}{2^{k}} \rightarrow 0 \text { as } k \rightarrow \infty .
$$

That is, $y_{k} \rightarrow p$. Since $F(S, T)$ is closed $(F(S)$ and $F(T)$ are closed by Lemma $2.1)$, therefore $p \in F(S, T)$.

In view of the inequality

$$
d\left(x_{n}, y_{k}\right) \leq \frac{\varepsilon_{k}}{4} \text { for all } n \geq N_{k},
$$

we get that $x_{n} \rightarrow p$. 
We extend Theorem 2.2 for three quasi-nonexpansive self mappings $R, S, T$ on $C$ by modifying (2) and Condition (A) in [16].

For any $x_{1} \in C$, we define a sequence $\left\{x_{n}\right\}$ by

$$
\begin{aligned}
x_{n+1} & =W\left(T y_{n}, W\left(S x_{n}, R x_{n}, \frac{\alpha_{n}^{(2)}}{1-\alpha_{n}^{(1)}}\right), \alpha_{n}^{(1)}\right), \\
y_{n} & =W\left(T x_{n}, W\left(S x_{n}, R x_{n}, \frac{\beta_{n}^{(2)}}{1-\beta_{n}^{(1)}}\right), \beta_{n}^{(1)}\right),
\end{aligned}
$$

where

$$
0<a \leq \alpha_{n}^{(1)} \leq b<1,0 \leq \beta_{n}^{(i)} \leq \beta<1 \text { for } i=1,2 .
$$

Three self mappings $S, T, R$ on $C$, satisfy Condition (II), if there exists a nondecreasing function $f:[0, \infty) \rightarrow[0, \infty)$ with $f(0)=0$ and $f(r)>0$ for $r>0$ such that

$$
d(T y, W(S x, R x, \alpha)) \geq f(d(x, F(S, T, R))), \alpha \in(0,1)
$$

for $x, y \in C$ with $y=W\left(T x, W\left(S x, R x, \frac{t}{1-s}\right), s\right)$ where $0 \leq s, t \leq \beta<1$ and $F(S, T, R)$ is the set of common fixed points of $S, T$ and $R$.

Theorem 2.3. Suppose $C$ is a closed, convex subset of a uniformly convex metric space $X$ satisfying property $(H)$. If $T, S$ and $R$ are quasi-nonexpansive self mappings on $C$ satisfying Condition (II), then the sequence (6) converges to an element of $F(S, T, R)$.

Proof. For $p \in F(S, T, R)$, we consider

$$
\begin{aligned}
d\left(x_{n+1}, p\right)= & d\left(W\left(T y_{n}, W\left(S x_{n}, R x_{n}, \frac{\alpha_{n}^{(2)}}{1-\alpha_{n}^{(1)}}\right), \alpha_{n}^{(1)}\right), p\right) \\
\leq & \alpha_{n}^{(1)} d\left(y_{n}, p\right)+\left(1-\alpha_{n}^{(1)}\right) d\left(W\left(S x_{n}, R x_{n}, \frac{\alpha_{n}^{(2)}}{1-\alpha_{n}^{(1)}}\right), p\right) \\
\leq & \alpha_{n}^{(1)} d\left(y_{n}, p\right)+\left(1-\alpha_{n}^{(1)}\right) d\left(x_{n}, p\right) \\
= & \alpha_{n}^{(1)} d\left(W\left(T x_{n}, W\left(S x_{n}, R x_{n}, \frac{\beta_{n}^{(2)}}{1-\beta_{n}^{(1)}}\right), \beta_{n}^{(1)}\right), p\right) \\
& +\left(1-\alpha_{n}^{(1)}\right) d\left(x_{n}, p\right) \\
\leq & \alpha_{n}^{(1)} d\left(x_{n}, p\right)+\left(1-\alpha_{n}^{(1)}\right) d\left(x_{n}, p\right) \\
= & d\left(x_{n}, p\right) .
\end{aligned}
$$


This gives that the sequences $\left\{d\left(x_{n}, p\right)\right\}$ and $\left\{d\left(x_{n}, F(S, T, R)\right)\right\}$ are nonincreasing and bounded below, therefore $\lim _{n \rightarrow \infty} d\left(x_{n}, p\right)$ and $\lim _{n \rightarrow \infty} d\left(x_{n}, F(S, T, R)\right)$ both exist.

Now we show that $\lim _{n \rightarrow \infty} d\left(x_{n}, F(S, T, R)\right)=0$.

Assume that $c=\lim _{n \rightarrow \infty} d\left(x_{n}, p\right)>0$.

Since

$$
\begin{aligned}
d\left(T y_{n}, p\right) \leq & d\left(y_{n}, p\right) \\
= & d\left(W\left(T x_{n}, W\left(S x_{n}, R x_{n}, \frac{\beta_{n}^{(2)}}{1-\beta_{n}^{(1)}}\right), \beta_{n}^{(1)}\right), p\right) \\
\leq & \beta_{n}^{(1)} d\left(T x_{n}, p\right)+\left(1-\beta_{n}^{(1)}\right) d\left(W\left(S x_{n}, R x_{n}, \frac{\beta_{n}^{(2)}}{1-\beta_{n}^{(1)}}\right), p\right) \\
& d\left(x_{n}, p\right)
\end{aligned}
$$

and

$$
\begin{aligned}
d\left(W\left(S x_{n}, R x_{n}, \frac{\alpha_{n}^{(2)}}{1-\alpha_{n}^{(1)}}\right), p\right) & \leq \frac{\alpha_{n}^{(2)}}{1-\alpha_{n}^{(1)}} d\left(S x_{n}, p\right)+\left(1-\frac{\alpha_{n}^{(2)}}{1-\alpha_{n}^{(1)}}\right) d\left(R x_{n}, p\right) \\
& \leq \frac{\alpha_{n}^{(2)}}{1-\alpha_{n}^{(1)}} d\left(x_{n}, p\right)+\left(1-\frac{\alpha_{n}^{(2)}}{1-\alpha_{n}^{(1)}}\right) d\left(x_{n}, p\right) \\
& \leq d\left(x_{n}, p\right)
\end{aligned}
$$

therefore

$$
\limsup _{n \rightarrow \infty} d\left(T y_{n}, p\right) \leq c, \limsup _{n \rightarrow \infty} d\left(W\left(S x_{n}, R x_{n}, \frac{\alpha_{n}^{(2)}}{1-\alpha_{n}^{(1)}}\right), p\right) \leq c .
$$

Also

$$
\lim _{n \rightarrow \infty} d\left(x_{n}, p\right)=c
$$

gives that

$$
\lim _{n \rightarrow \infty} d\left(W\left(T y_{n}, W\left(S x_{n}, R x_{n}, \frac{\alpha_{n}^{(2)}}{1-\alpha_{n}^{(1)}}\right), \alpha_{n}^{(1)}\right), p\right)=c
$$

In the light of (7) and (8), we set $u_{n}=T y_{n}, v_{n}=W\left(S x_{n}, R x_{n}, \frac{\alpha_{n}^{(2)}}{1-\alpha_{n}^{(1)}}\right), \alpha_{n}=$ $\alpha_{n}^{(1)}$ in Lemma 1.5 and conclude that

$$
\lim _{n \rightarrow \infty} d\left(T y_{n}, W\left(S x_{n}, R x_{n}, \frac{\alpha_{n}^{(2)}}{1-\alpha_{n}^{(1)}}\right)\right)=0 .
$$


Further, by condition (II), we get $\lim _{n \rightarrow \infty} d\left(x_{n}, F(S, T, R)\right)=0$.

The rest of the proof is similar to the proof in Theorem 2.2.

Using the concept of unique point $(1-\alpha) x \oplus \alpha y$ in the geodesic segment $[x, y]$ from the point $x$ to the point $y$ in $C A T(0)$ space $X$, one can see that $W(x, y, \alpha)=(1-\alpha) x \oplus \alpha y$ is a convex structure in $X$. Therefore, Theorems 2.2- 2.3 are valid in $C A T(0)$ space and are formulated as follows:

Theorem 2.4. Let $C$ be a nonempty, closed and convex subset of a $C A T(0)$ space. For two quasi-nonexpansive self mappings $S$ and $T$ on $C$,define $\left\{x_{n}\right\}$ by

$$
\begin{aligned}
x_{n+1} & =\alpha_{n} T y_{n} \oplus\left(1-\alpha_{n}\right) S x_{n} \\
y_{n} & =\beta_{n} T x_{n} \oplus\left(1-\beta_{n}\right) S x_{n} \text { for } n \geq 1 .
\end{aligned}
$$

If $S$ and $T$ satisfy Condition (I), then $\left\{x_{n}\right\}$ converges to a common fixed point of $S$ and $T$.

Theorem 2.5. Let $C$ be a nonempty, closed and convex subset of a $C A T(0)$ space. For three quasi-nonexpansive self mappings $T, S$ and $R$ on $C$, define $\left\{x_{n}\right\}$ by

$$
\begin{aligned}
x_{n+1} & =\alpha_{n}^{(1)} T y_{n} \oplus\left(1-\alpha_{n}^{(1)}\right)\left[\frac{\alpha_{n}^{(2)}}{1-\alpha_{n}^{(1)}} S x_{n} \oplus\left(1-\frac{\alpha_{n}^{(2)}}{1-\alpha_{n}^{(1)}}\right) R x_{n}\right] \\
y_{n} & =\beta_{n}^{(1)} T x_{n} \oplus\left(1-\beta_{n}^{(1)}\right)\left[\frac{\beta_{n}^{(2)}}{1-\beta_{n}^{(1)}} S x_{n} \oplus\left(1-\frac{\beta_{n}^{(2)}}{1-\beta_{n}^{(1)}} R x_{n}\right)\right], n \geq 1 .
\end{aligned}
$$

If $0<a \leq \alpha_{n}^{(1)} \leq b<1,0 \leq \beta_{n}^{(i)} \leq \beta<1$ for $i=1,2$ and the mappings $R, S$ and $T$ satisfy Condition (II), then $\left\{x_{n}\right\}$ converges to a common fixed point of $R, S$ and $T$.

Acknowledgment. The author is grateful to King Fahd University of Petroleum $\&$ Minerals for supporting research project IN121055.

\section{References}

[1] V. Berinde, Iterative Approximation of Fixed Points, Springer, Berlin Heidelberg New York, 2007

[2] M. Bridson and A. Haefliger, Metric spaces of non-positive curvature, Springer-Verlag, Berlin, Heidelberg, New York, 1999.

[3] H. Busemann, Spaces with non-positive curvature, Acta Math. 80 (1948), 259-310. 
[4] Cegielski, A., Iterative methods for fixed point problems in Hilbert spaces, Lecture Notes in Mathematics, 2057, Springer, Heidelberg, 2012.

[5] Chidume, C. E., Geometric Properties of Banach Spaces and Nonlinear Iteration, Springer, Berlin Heidelberg New York, 2009.

[6] G. Das and P. Debata, Fixed points of quasi-nonexpansive mappings, Indian J. Pure Appl. Math. 17(1986), 1263-1269.

[7] H. Fukhar-ud-din, A. R. Khan and Z. Akhtar, Fixed point results for a generalized nonexpansive map in uniformly convex metric spaces, Nonlinear Anal., 75 (2012), 4747-4760.

[8] M. K. Ghosh and L. Debnath, Convergence of Ishikawa iterates of quasinonexpansive mappings, J. Math. Anal. Appl., 207 (1997), 96-103.

[9] S. Ishikawa, Fixed points by a new iteration method, Proc. Amer. Math. Soc., 44 (1974), 147-150.

[10] S. H. Khan and H. Fukhar-ud-din, Weak and strong convergence of a scheme with errors for two nonexpansive mappings, Nonlinear Anal., 61 (2005), $1295-1301$.

[11] A. R. Khan, H. Fukhar-ud-din and M. A. A. Khan, An implicit algorithm for two finite families of nonexpansive maps in hyperbolic spaces, Fixed Point Theory and Applications, 2012, 2012:54 (doi:10.1186/1687-18122012-54).

[12] A. R. Khan, H. Fukhar-ud-din and A.A. Domlo, Approximating fixed points of some maps in uniformly convex metric spaces, Fixed Point Theory Applications, 2010 (2010) Article ID 385986, 11 pages.

[13] A. R. Khan, M. A. Khamsi and H. Fukhar-ud-din, Strong convergence of a general iteration scheme in CAT(0)-spaces, Nonlinear Anal. 74 (2011), 783-791.

[14] W. A. Kirk, An abstract fixed point theorem for nonexpansive mappings, Proc. Amer. Math. Soc., 82(1981), 640-642.

[15] W. A. Kirk, Fixed point theory for nonexpansive mappings II, Contemporary Math.,18(1983), 121-140.

[16] M. Maiti and M. K. Ghosh, Approximating fixed points by Ishikawa iterates, Bull. Austral. Math. Soc. 40 (1989), 113-I 17. 
CONVERGENCE OF ISHIKAWA TYPE ITERATION PROCESS FOR THREE

[17] W. R. Mann, Mean value methods in iterations, Proc. Amer. Math. Soc. 4 (1953), 506-510.

[18] J. P. Penot, Fixed point theorems without convexity, Bull. Soc. Math. France, 60(1979), 129-152.

[19] T. Shimizu and W. Takahashi, Fixed points of multivalued mappings in certain convex metric spaces, Topol. Methods Nonlinear Anal. 8 (1996), 197-203.

[20] W. Takahashi, A convexity in metric spaces and nonexpansive mappings, Kodai. Math. Sem. Rep., 22(1970), 142-149.

Hafiz Fukhar-ud-din,

Department of Mathematics and Statistics,

King Fahd University of Petroleum and Minerals,

Dhahran 31261, Saudi Arabia;

Department of Mathematics,

The Islamia University of Bahawlpur 63100, Pakistan

Email: hfdin@kfupm.edu.sa ; hfdin@yahoo.com 